\title{
Interacting Kasner-type cosmologies
}

\author{
Mauricio Cataldo ${ }^{1}$ - Fabiola Arévalo ${ }^{2}$ • \\ Patricio Mella ${ }^{3}$
}

\begin{abstract}
It is well known that Kasner-type cosmologies provide a useful framework for analyzing the threedimensional anisotropic expansion because of the simplification of the anisotropic dynamics. In this paper relativistic multi-fluid Kasner-type scenarios are studied. We first consider the general case of a superposition of two ideal cosmic fluids, as well as the particular cases of non-interacting and interacting ones, by introducing a phenomenological coupling function $q(t)$. For two-fluid cosmological scenarios there exist only cosmological scaling solutions, while for three-fluid configurations there exist not only cosmological scaling ones, but also more general solutions. In the case of triply interacting cosmic fluids we can have energy transfer from two fluids to a third one, or energy transfer from one cosmic fluid to the other two. It is shown that by requiring the positivity of energy densities there always is a matter component which violates the dominant energy condition in this kind of anisotropic cosmological scenarios.
\end{abstract}

Keywords Kasner-type cosmologies

Mauricio Cataldo

Departamento de Física, Facultad de Ciencias, Universidad del Bío-Bío, Avenida Collao 1202, Casilla 5-C, Concepción, Chile.

\section{Fabiola Arévalo}

Departamento de Física, Universidad de Concepción, Casilla 160-C, Concepción, Chile.

\section{Patricio Mella}

Departamento de Física, Universidad de Concepción, Casilla 160-C, Concepción, Chile.

${ }^{1}$ mcataldo@ubiobio.cl

${ }^{2}$ farevalo@udec.cl

${ }^{3}$ patriciomella@udec.cl

\section{Introduction}

It is well known that the present cosmological observations allow the theoretical cosmology, based on General Relativity, to conclude that nearly $96 \%$ of the matter content in the Universe is of types which have not been seen in the laboratory. Even more, there is also indirect evidence suggesting that nearly $74 \%$ of the matter content present in the Universe exerts a negative pressure (dark energy). So one of the key problems of the current cosmology is deciding which kinds of matter sources were and are present in the Universe. This notable uncertainty about the kind of matter filling the Universe, which is unique when we attempt to apply the laws of physics to the evolution of the Universe, requires the cosmologists to proceed in a multi-faceted manner (Padmanabhan 2005).

Usually cosmological models are constructed under the assumption that the matter source is an idealized perfect fluid. This assumption may be a good approximation to the matter content of the Universe, however at earlier epochs there may not be so negligible the effects of anisotropic matter fields such as for example magnetic and electric fields, populations of collisionless particles such as gravitons, photons or relativistic neutrinos; long-wavelength gravitational waves, topological defects such as cosmic string and domain walls, among others (Barrow 1997,1999).

On the other hand, in recent years the current Cosmology has been strongly influenced by the huge improvement in quality, quantity and the scope of cosmological observations (Page et al 2006; Astier et al 2006; Tegmark et al 2004; Bennett et al 2003; Hinshaw et al 2006; Percival et al 2006,2007; (Spergel et al 2003,2006). Recent investigations detect anisotropy in the cosmic microwave background radiation (CMBR). The temperature anisotropy in the CMBR has been arguably the 
most influential of these recent cosmological observations and represents one of the most accurate observational data in the modern cosmology. This scenario leads cosmologists to consider for early stages of the Universe not only the standard isotropic and homogeneous FRW metrics but also to consider more general universe models. In principle one can consider inhomogeneous models which, in order to reproduce the late homogenous and isotropic stages of the Universe, must contain a FRW background for certain limits or values of the model functions or parameters respectively. On the other hand one can also consider anisotropic backgrounds. The simplest generalization of FRW spacetimes are the homogeneous and anisotropic Bianchi cosmologies of types IX, V and I, which are generalizations of closed, open and flat FRW spacetimes respectively.

In the latter Bianchi type I we find the special class of Kasner-type geometries, for which cosmological scale factors evolve as a power law in time. It is important to notice that Kasner-type geometries themselves have been used as a simple arena for discussing some properties of anisotropically expanding cosmologies. It is very natural for anisotropic Kasner-type cosmologies to introduce a matter source described by an imperfect fluid with a stress-energy tensor containing the coefficients of bulk viscosity, shear viscosity and heat conduction. In Refs. Brevik 1997; Cataldo et al 2000; Brevik et al 2000) are discussed anisotropic matter sources for this kind of cosmologies. It was shown that in general relativity the Bianchi type I metric of the Kasner form is not able to describe an anisotropic universe filled with a viscous fluid, satisfying simultaneously the dominant energy condition (DEC) and the second law of thermodynamics (Cataldo et al 2000). In Ref. Cataldo et al 2001) it is proved that this is possible in scalar tensor theories, while for a more general Bianchi type I metric with a perfect fluid it is possible in the Brans-Dicke theory of gravity (Lee 2008).

On the other hand, in Ref. Cataldo et al 2001) the discussion goes into the framework of the holographic principle, in Ref. Halpern 2001) the behavior of Kasner-type Cosmologies with Induced Matter is studied, while in other more general and interesting contexts Kasner geometries also are considered (Ponce de Leon 2009; Svitek 2006; Copeland et al 2010; Mak et al 2002; Bini et al 2007; Ivashchuk et al 2008). Lastly, let us note that the Kasner metric plays an important role also in Bianchi cosmologies of type IX (the Mixmaster Universe), where in vacuum solutions the Mixmaster universe has served as a theoretical playground for many ideas related to the question of the nature of the chaotic behavior exhibited in some solutions of the vacuum Einstein equations (Bini et al 2009), or for models containing matter where the spatial curvature causes the axes and rates of contraction to undergo sudden jumps from one Kasner-like solution to another (Erickson et al 2004). The Kasner spacetimes are often used for the description of the very early stages of the Universe.

In this paper we shall consider Kasner-type cosmologies dominated by two or three matter components. The interaction among ideal fluid components also will be considered. The interest in interacting cosmologies has been focused mainly on FRW cosmologies in order to address the observed late acceleration of the Universe (Armendariz-Picon et al 2000; Bean et al 2001; Riess et al 2004; Srivastava 2006) and the so called cosmological coincidence problem (Cataldo et al 2008; Guo et al 2005; Pavon et al 2005; Chimento et al 2003). Usually the universe is modeled with perfect fluids and with mixtures of non-interacting perfect fluids. This means that it is assumed that there is no conversion (energy transfer) among the components and that each of them evolves separately according to standard conservation laws. However, we can consider plausible cosmological models containing fluids which interact with each other, so the energy from one of the fluids is diluted or decayed into another fluid component. The advantage of considering multi-fluid components in anisotropic Kasner-type cosmologies is that the problem is solved exactly, in an analytical manner.

The outline of the present paper is as follows: In Section II we establish and solve the Einstein field equations for two-fluid Kasner-type cosmologies. In Section III solutions for three-fluid configurations are considered. Finally, Section IV presents some concluding remarks.

\section{Einstein field equations for two-fluid Kasner-type cosmologies}

We shall consider in this paper anisotropic cosmologies described by the metric

$d s^{2}=d t^{2}-t^{2 p_{1}} d x^{2}-t^{2 p_{2}} d y^{2}-t^{2 p_{3}} d z^{2}$,

where we shall call $p_{1}, p_{2}$ and $p_{3}$ Kasner parameters and they are constant ones. We shall refer to the Kasnertype cosmologies (1) as simply Kasner cosmologies.

Thus, the Einsteins field equations $R_{\mu \nu}-\frac{1}{2} R g_{\mu \nu}=$ $-\kappa T_{\mu \nu}$ reduce to

$\kappa \rho=\frac{p_{1} p_{2}+p_{1} p_{3}+p_{2} p_{3}}{t^{2}}$, 


$$
\begin{aligned}
& \kappa P_{x}=-\frac{p_{2}^{2}+p_{3}^{2}-p_{2}-p_{3}+p_{2} p_{3}}{t^{2}} \\
& \kappa P_{y}=-\frac{p_{1}^{2}+p_{3}^{2}-p_{1}-p_{3}+p_{1} p_{3}}{t^{2}} \\
& \kappa P_{z}=-\frac{p_{1}^{2}+p_{2}^{2}-p_{1}-p_{2}+p_{1} p_{2}}{t^{2}}
\end{aligned}
$$

Here $\kappa=8 \pi G$ and $P_{j}$, with $j=x, y, z$, representing the effective momenta in the corresponding coordinate axis, implying that in general for a Kasner metric we have anisotropic pressures. In other words, for the metric (11) one might introduce a matter source described by an imperfect fluid. Thus for a Kasner space-time one might consider a stress-energy tensor of an imperfect fluid containing the coefficients of bulk viscosity, shear viscosity and heat conduction. Note that in this case the energy density and all pressures scale as $1 / t^{2}$, implying that we have barotropic equations of state for all pressures, i.e. $P_{j}=\omega_{j} \rho$, with $\omega_{j}$ constants. However, in this paper we shall consider only perfect cosmic fluids, i.e. $P_{x}=P_{y}=P_{z}$. Note that in this case either $\rho$ and $P_{j}$ (with $j=x, y, z$ ) scale always as $t^{-2}$.

Let us now consider a Kasner cosmology filled with two ideal cosmic fluids $\rho_{1}$ and $\rho_{2}$. Thus by putting into Eqs.(2)-(5) the expressions $\rho=\rho_{1}+\rho_{2}$ and $P_{x}=P_{y}=$ $P_{z}=P_{1}+P_{2}$ we may rewrite the field equations in the following form:

$$
\begin{aligned}
S-Q & =\frac{1}{2} \kappa t^{2}\left(\rho_{1}+\rho_{2}+3\left(P_{1}+P_{2}\right)\right), \\
p_{i}(1-S) & =-\frac{1}{2} \kappa t^{2}\left(\rho_{1}+\rho_{2}-\left(P_{1}+P_{2}\right)\right),
\end{aligned}
$$

where we have introduced

$$
S=p_{1}+p_{2}+p_{3},
$$$$
Q=p_{1}^{2}+p_{2}^{2}+p_{3}^{2} \text {, }
$$

and $i=1,2,3$.

Note that we obtain the same equations if we write the Einstein equations in the form $R_{\mu \nu}=-\kappa\left(T_{\mu \nu}+\right.$ $\left.T g_{\mu \nu}\right)$. It is easy to see that for the vacuum Kasner solution we have $S=Q=1$.

\subsection{General solution for two-fluid Kasner cosmologies}

We are interested in studying cosmological scenarios filled with two cosmic fluids which have barotropic equation of state

$$
\begin{aligned}
& P_{1}(t)=\omega_{1} \rho_{1}(t), \\
& P_{2}(t)=\omega_{1} \rho_{2}(t),
\end{aligned}
$$

with $\omega_{1}$ and $\omega_{2}$ constants. Let us now consider the direct integration of the field equations (6) and (7).
From three Eqs. (7) we conclude that, in order to satisfy all these equations with self-consistent expressions for energy densities and pressures $\rho_{1}, \rho_{2}, P_{1}$ and $P_{2}$, we must impose the condition $S=1$. This implies that $\rho_{1}+\rho_{2}=P_{1}+P_{2}$, and then

$\rho_{1}+\rho_{2}=\omega_{1} \rho_{1}+\omega_{2} \rho_{2}$.

Thus by taking into account Eq $[$ we obtain for energy densities

$\begin{aligned} \kappa \rho_{1} & =\frac{(1-Q)\left(1-\omega_{2}\right)}{2\left(\omega_{1}-\omega_{2}\right) t^{2}}, \\ \kappa \rho_{2} & =\frac{(1-Q)\left(\omega_{1}-1\right)}{2\left(\omega_{1}-\omega_{2}\right) t^{2}},\end{aligned}$

where $\omega_{1} \neq \omega_{2}$. For $\omega_{1}=\omega_{2}$ we obtain the trivial case of a single fluid in a Kasner cosmology.

Since we are interested in a characterization of twofluid Kasner cosmologies, we shall consider that the weak energy condition (WEC) holds and then we shall require for each source component that $\rho_{1} \geq 0$ and $\rho_{2} \geq 0$. These conditions will imply that

$0 \leq Q \leq 1$

for $\omega_{2} \leq 1, \omega_{1} \geq 1$ or $\omega_{2} \geq 1, \omega_{1} \leq 1$. Thus the condition (12) still holds as in the case of a Kasner cosmology filled with a single ideal fluid.

Note that one of the fluids, say $\rho_{1}$, can satisfy the DEC (i.e. $-1 \leq \omega_{1} \leq 1$ ) or have a phantom behavior $\left(\omega_{1}<-1\right)$, while another component, say $\rho_{2}$, has a state parameter satisfying $\omega_{2}>1$, thus always violating DEC.

\subsection{Non-interacting cosmic fluids}

Usually the Universe is modeled with single perfect fluids and with mixtures of non-interacting perfect fluids Gunzig et al 2000; Pinto-Neto 2005; Bozza et al 2005; Goliath et al 2000; Gavrilov et al 2004). This means that it is assumed that there is no conversion (energy transfer) among the components and that each of them evolves separately. Now in order to study the following two cases for the superposition of two cosmic fluids we shall use the conservation equation for Kasner cosmologies, which is given by

$\dot{\rho}_{1}+\dot{\rho}_{2}+\frac{S}{t}\left(\rho_{1}+\rho_{2}+P_{1}+P_{2}\right)=0$.

Thus for two non-interacting perfect fluids we can write the standard conservation laws

$\dot{\rho}_{1}+\frac{S}{t}\left(\rho_{1}+P_{1}\right)=0$,
$\dot{\rho}_{2}+\frac{S}{t}\left(\rho_{2}+P_{2}\right)=0$, 
which identically satisfy the general conservation equation (13). By taking into account the barotropic equations of state (9) both of these equations can easily be integrated obtaining

$\rho_{i}(t)=\frac{C_{i}}{t^{S\left(1+\omega_{i}\right)}}$,

with $i=1,2$. Now, since the total energy density $\rho$ scale as $1 / t^{2}$ we have that

$\frac{C_{1}}{t^{S\left(1+\omega_{1}\right)}}+\frac{C_{2}}{t^{S\left(1+\omega_{2}\right)}} \sim t^{-2}$

This implies that the condition $S\left(1+\omega_{1}\right)=S\left(1+\omega_{2}\right)=$ 2 must be imposed, leading finally to $\omega_{1}=\omega_{2}$. Thus both fluids have the same state equation and then the non-interacting superposition of two fluids is trivially equivalent to the standard scenario with a single cosmic fluid.

\subsection{Interacting cosmic fluids}

In this case we can rewrite the conservation equation (13) in the following form:

$\dot{\rho}_{1}+\frac{S}{t}\left(\rho_{1}+P_{1}\right)=q(t)$,

$\dot{\rho}_{2}+\frac{S}{t}\left(\rho_{2}+P_{2}\right)=-q(t)$,

where is introduced a phenomenological coupling function $q(t)$. Such a kind of interacting term has been considered before in the literature (del Campo et al 2006; Tomaras 2006; Barrow et al 2006; Kofinas et al 2006). Note that if $q(t)>0$ we have that there exists a transfer of energy from the fluid $\rho_{2}$ to the fluid $\rho_{1}$. Again the general conservation equation (13) is identically satisfied.

Now by considering barotropic equations of state (9) we conclude that the interaction term has the general form $q(t)=q_{0} / t^{3}$, where $q_{0}$ is an arbitrary constant. Thus by putting $q(t)=q_{0} / t^{3}$ into Eqs.(17) and (18) we obtain the following solutions for the energy densities:

$\rho_{i}(t)= \pm \frac{C_{i}}{t^{S\left(1+\omega_{i}\right)}}+\frac{q_{0}}{\left(S\left(1+\omega_{i}\right)-2\right) t^{2}}$,

where $i=1,2 ; C_{i}$ are integration constants and the positive (negative) sign corresponds to $i=1(i=2)$. Note that due to the fact that in Kasner cosmologies the total energy density $\rho$ scales as $t^{-2}$, without any loss of generality, we can put $C_{i}=0\left(\omega_{1} \neq \omega_{2}\right)$.

Now, since the pressures are isotropic, from Eq.(7) we obtain that $S=1$. Then by putting the expressions for energy densities $\rho_{1}$ and $\rho_{2}$ from Eq. (19) (with $C_{1}=$ $C_{2}=0$ and $S=1$ ) into Eq. (6) we obtain

$q_{0}=\frac{(1-Q)\left(\omega_{1}-1\right)\left(1-\omega_{2}\right)}{2 \kappa\left(\omega_{1}-\omega_{2}\right)}$,

leading to the same expressions for energy densities (10) and (11) of Section II-A. It is interesting to note that the interaction term is characterized by a coupling of the form

$q(t)=\left(\omega_{1}-1\right) \theta \rho_{1}=\left(1-\omega_{2}\right) \theta \rho_{2}$,

where $\theta$ is the expansion factor and in general for the metric (1) is given by $\theta=S / t$. Notice that interacting cosmological models with the interaction of the form (21) were considered in the framework of flat FRW cosmologies (Olivares et al 2008; Zimdahl et al 2001; Setare et al 2009; Sadjadi e al 2006; Nojiri et al 2006; Rashid et al 2009).

It is clear from conditions $\rho_{1} \geq 0$ and $\rho_{2} \geq 0$ that we have the same constraint (12) on $Q$. Thus from Eq.(12) and $\omega_{2} \leq 1, \omega_{1} \geq 1$ we obtain that $q(t) \geq 0$ so we have energy transfer from $\rho_{2}$ to fluid $\rho_{1}$; and from Eq.(12) and $\omega_{2} \geq 1, \omega_{1} \leq 1$ we have that $q(t) \leq 0$ so we have energy transfer from $\rho_{1}$ to the cosmic fluid $\rho_{2}$.

It calls to attention that the energy densities for the interacting case coincide with the energy densities of the subsection II-A. Mainly this is due to the power law character of scale factors in the Kasner metric (1). In order to see this more clearly let us discuss the general solution obtained in Section II-A. In principle, on general expressions (10)-111) we can now impose particular requirements. For example let us impose the condition (14). Thus we obtain the following constraints on state parameters: $\omega_{1}=\omega_{2}=1$. This implies that we really have a single stiff fluid filling the Kasner cosmology. On the other hand, if we now require that both cosmic fluids (10)-111) interact with each other, then the fulfillment of the conditions (17)-18) does not add any extra condition on the model parameters $\omega_{1}$, $\omega_{2}$ and $Q$, defining only the final form of the interacting term $q(t)=q_{0} / t^{3}$ (with $q_{0}$ expressed by Eq.(20) ). Thus the form of the energy densities (10)-111) remains unchanged. However, we can impose an explicit form on $q(t)$. For example, for interacting terms given by $q(t)=\alpha \theta \rho_{1}, q(t)=\beta \theta \rho_{2}$ or $q(t)=\gamma \theta \rho_{1}+\delta \theta \rho_{2}$ we shall obtain some constraints on $\omega_{1}, \omega_{2}, Q$ with constant parameters $\alpha, \beta$ or $\gamma$ and $\delta$ respectively.

\section{Three-fluid Kasner cosmologies}

In the following we shall consider Kasner cosmologies filled with three barotropic cosmic fluids $\rho_{1}, \rho_{2}$ and $\rho_{3}$. 
It is clear that the field equations now are given by

$$
\begin{aligned}
S-Q & =\frac{1}{2} \kappa t^{2}\left(\rho_{1}+\rho_{2}+\rho_{3}+3\left(P_{1}+P_{2}+P_{3}\right)\right), \\
p_{i}(1-S) & =-\frac{1}{2} \kappa t^{2}\left(\rho_{1}+\rho_{2}+\rho_{3}-\left(P_{1}+P_{2}+P_{3}\right)\right),
\end{aligned}
$$

where $i=1,2,3$. Now we shall consider a barotropic equation of state $P_{i}=\omega_{i} \rho_{i}$ for each fluid. Since there are two differential equations with three unknown functions $\rho_{i}$, we shall suppose $\rho_{3}$ as a given function, in order to close the system of equations.

Requiring that $S=1$, from Eq. (23) we obtain that

$\rho_{1}\left(1-\omega_{1}\right)+\rho_{2}\left(1-\omega_{2}\right)+\rho_{3}\left(1-\omega_{3}\right)=0$.

Solving Eqs.(22) and (24) we obtain

$\kappa \rho_{1}=\frac{(1-Q)\left(1-\omega_{2}\right)}{2\left(\omega_{1}-\omega_{2}\right) t^{2}}+\frac{\omega_{2}-\omega_{3}}{\omega_{1}-\omega_{2}} \kappa \rho_{3}$,
$\kappa \rho_{2}=\frac{(1-Q)\left(\omega_{1}-1\right)}{2\left(\omega_{1}-\omega_{2}\right) t^{2}}+\frac{\omega_{3}-\omega_{1}}{\omega_{1}-\omega_{2}} \kappa \rho_{3}$.

Let us now elucidate if all state parameters will respect DEC when the fulfillment of WEC is required, i.e. $\rho_{i} \geq 0$ (for each $i=1,2,3$ ). By adding Eqs. (25) and (26) and taking into account condition $\rho_{3} \geq 0$ we conclude that the constrain (12) is valid. In order to have different equations of state for the cosmic ideal fluids we must require $\omega_{1} \neq \omega_{2} \neq \omega_{3}$. This implies that, without any loss of generality, we can put $\omega_{3}<\omega_{2}<\omega_{1}$. Thus from Eqs.(26) and (12) we conclude that we must require that $\omega_{1}>1$ in order to fulfill the requirement $\rho_{2} \geq 0$. Thus, always at least one of the three state parameters violates DEC, implying that in three-fluid Kasner cosmologies it is not possible to have simultaneously, for each fluid, $\omega_{i}<1$ if we require that $\rho_{i} \geq 0$ $(i=1,2,3)$. This leads to the impossibility of having all three fluids simultaneously satisfying DEC, i.e. $-1 \leq \omega_{i} \leq 1$.

Note that for this general solution we can invoke the conservation equation, which is identically satisfied by energy densities (25) and (26), and has the following form:

$$
\begin{aligned}
& \dot{\rho}_{1}+\dot{\rho}_{2}+\dot{\rho}_{3} \\
& +\frac{1}{t}\left(\rho_{1}\left(1+\omega_{1}\right)+\rho_{2}\left(1+\omega_{2}\right)+\rho_{3}\left(1+\omega_{3}\right)\right)=0 .
\end{aligned}
$$

Clearly, one can consider the case where a two-fluid configuration is conserved separately from a third component, i.e. we have

$$
\begin{aligned}
\dot{\rho}_{1}+\dot{\rho}_{2}+\frac{1}{t}\left(\rho_{1}\left(1+\omega_{1}\right)+\rho_{2}\left(1+\omega_{2}\right)\right) & =0 \\
\dot{\rho}_{3}+\frac{1}{t}\left(1+\omega_{3}\right) & =0 .
\end{aligned}
$$

Of course this is a particular solution of the above obtained general solution. From Eq.(29) we obtain that

$\rho_{3}(t)=C t^{-\left(1+\omega_{3}\right)}$,

where $C$ is an integration constant. Thus the solution in this case takes the form

$\kappa \rho_{1}=\frac{(1-Q)\left(1-\omega_{2}\right)}{2\left(\omega_{1}-\omega_{2}\right) t^{2}}+\frac{\kappa C\left(\omega_{2}-\omega_{3}\right)}{\left(\omega_{1}-\omega_{2}\right) t^{1+\omega_{3}}}$,
$\kappa \rho_{2}=\frac{(1-Q)\left(\omega_{1}-1\right)}{2\left(\omega_{1}-\omega_{2}\right) t^{2}}+\frac{\kappa C\left(\omega_{3}-\omega_{1}\right)}{\left(\omega_{1}-\omega_{2}\right) t^{1+\omega_{3}}}$.

Such a solution may describe multi-fluid Kasner cosmologies filled with a two-fluid conserved configuration and independently conserved dust $\left(\omega_{3}=0\right)$, radiation $\left(\omega_{3}=1 / 3\right)$, or even a cosmological constant $\left(\omega_{3}=-1\right)$, among others. If the third fluid is a stiff one, i.e. $\omega_{3}=1$, this solution becomes a scaling cosmological solution. Note that for the case where each fluid component is conserved separately we obtain that $\rho_{3}(t)=\frac{1-Q}{2 \kappa t^{2}}$ with $\rho_{1}=\rho_{2}=0$, so this case is equivalent to the trivial scenario of a single stiff fluid in a Kasner cosmology.

Now let us consider interacting scenarios for these three fluids $\rho_{1}, \rho_{2}$ and $\rho_{3}$. We can thus write (where $S=1$ and $\left.P_{i}=\omega_{i} \rho_{i}\right)$

$\dot{\rho}_{1}+\frac{\rho_{1}}{t}\left(1+\omega_{1}\right)=\alpha(t)$,

$\dot{\rho}_{2}+\frac{\rho_{2}}{t}\left(1+\omega_{2}\right)=\beta(t)$,

$\dot{\rho}_{3}+\frac{\rho_{3}}{t}\left(1+\omega_{3}\right)=\gamma(t)$,

where $\alpha, \beta$ and $\gamma$ must be constrained as follows:

$\alpha(t)+\beta(t)+\gamma(t)=0$.

By introducing into Eqs.(32) and (33) the general expressions for energy densities (25) and (26) we obtain

$$
\begin{aligned}
\alpha(t)= & \frac{(1-Q)\left(\omega_{1}-1\right)\left(1-\omega_{2}\right)}{2 \kappa\left(\omega_{1}-\omega_{2}\right) t^{3}} \\
& +\frac{\left(1+\omega_{1}\right)\left(\omega_{2}-\omega_{3}\right)}{\left(\omega_{1}-\omega_{2}\right) t} \rho_{3}(t)+\frac{\omega_{2}-\omega_{3}}{\omega_{1}-\omega_{2}} \dot{\rho}_{3}, \\
\beta(t)= & -\frac{(1-Q)\left(\omega_{1}-1\right)\left(1-\omega_{2}\right)}{2 \kappa\left(\omega_{1}-\omega_{2}\right) t^{3}} \\
& -\frac{\left(1+\omega_{2}\right)\left(\omega_{1}-\omega_{3}\right)}{\left(\omega_{1}-\omega_{2}\right) t} \rho_{3}(t)+\frac{\omega_{3}-\omega_{1}}{\omega_{1}-\omega_{2}} \dot{\rho}_{3} .
\end{aligned}
$$

Since Eqs. (25) and (26) are the general expressions for energy densities in Kasner cosmologies filled with relativistic ideal fluids $\rho_{1}, \rho_{2}$ and $\rho_{3}$, the substitution of Eqs.(25) and (26) into Eqs.(32)-(34) yields the identical fulfillment of constraint (35). Thus, in order to consider 
different interacting scenarios we can impose a specific form on the interacting term $\gamma(t)$, and then, by using Eqs. (36) and (37), find interacting terms $\alpha(t)$ and $\beta(t)$ and the final forms for energy densities (25) and (26).

Let us for example consider the scenario where $\rho_{1}$ and $\rho_{2}$ are interacting with each other while $\rho_{3}$ is conserved separately. Such a solution may describe a multifluid Kasner cosmology filled with two interacting fluids and a conserved dust $\left(\omega_{3}=0\right)$, radiation $\left(\omega_{3}=1 / 3\right)$ or cosmological constant $\left(\omega_{3}=-1\right)$, among others. This means that we must put $\gamma(t)=0$, obtaining from Eq. (34) that the energy density $\rho_{3}(t)$ is given by Eq. (30). In this case Eq. (35) becomes $\alpha+\beta=0$. For $C=0$ we obtain the case of two interacting fluids discussed in Section II. Thus the general solution in this case takes the form (31) with interaction terms given by

$$
\begin{array}{r}
\alpha(t)=\frac{(1-Q)\left(\omega_{1}-1\right)\left(1-\omega_{2}\right)}{2 \kappa\left(\omega_{1}-\omega_{2}\right) t^{3}} \\
+\frac{C\left(\omega_{1}-\omega_{3}\right)\left(\omega_{2}-\omega_{3}\right)}{\left(\omega_{1}-\omega_{2}\right) t^{2+\omega_{3}}}=-\beta(t) .
\end{array}
$$

Now, as another example, we shall consider a scenario where all three fluids interact with each other. Let us suppose that

$\gamma(t)=\frac{q_{30}}{t^{n}}$

where $q_{30}$ and $n$ are constants. Thus from Eq. (34) we obtain that the energy density of the third fluid is given by

$\rho_{3}=\frac{q_{30}}{\left(-n+2+\omega_{3}\right) t^{n-1}}+C t^{-\left(1+\omega_{3}\right)}$,

which helps us to find the form of energy densities $\rho_{1}$ and $\rho_{2}$ by Eqs. 25) and (26). Thus other interacting terms are given by

$$
\begin{aligned}
\alpha(t)= & \frac{\left(\omega_{2}-\omega_{3}\right)\left(\omega_{1}+2-n\right) q_{30}}{\left(\omega_{3}-n+2\right)\left(\omega_{1}-\omega_{2}\right) t^{n}} \\
& +\frac{(1-Q)\left(\omega_{1}-1\right)\left(1-\omega_{2}\right)}{2 \kappa\left(\omega_{1}-\omega_{2}\right) t^{3}} \\
& +\frac{C\left(\omega_{1}-\omega_{3}\right)\left(\omega_{2}-\omega_{3}\right)}{\left(\omega_{1}-\omega_{2}\right) t^{2+\omega_{3}}}, \\
\beta(t)= & \frac{\left(\omega_{3}-\omega_{1}\right)\left(\omega_{2}-n+2\right) q_{30}}{\left(\omega_{3}-n+2\right)\left(\omega_{1}-\omega_{2}\right) t^{3}} \\
& -\frac{(1-Q)\left(\omega_{1}-1\right)\left(1-\omega_{2}\right)}{2 \kappa\left(\omega_{1}-\omega_{2}\right) t^{3}} \\
& -\frac{C\left(\omega_{1}-\omega_{3}\right)\left(\omega_{2}-\omega_{3}\right)}{\left(\omega_{1}-\omega_{2}\right) t^{2+\omega_{3}} .}
\end{aligned}
$$

The interacting term given by Eq. (39) generalizes the kind of interacting term discussed in Section II-C for which, to obtain it we must put $n=3$. In this case, if additionally we have $\omega_{3}=1$, this solution becomes a scaling cosmological solution. For $q_{30}=0$ we obtain the previously discussed case of a multi-fluid Kasner cosmology filled with two interacting fluids and a single conserved one.

Since now we have three interacting terms, it is possible to have two of them positive and one negative, or two negatives and one positive. For example suppose that $\gamma<0, \alpha>0$ and $\beta>0$. This implies that we have energy transfer from the third fluid $\rho_{3}$ to the fluids $\rho_{1}$ and $\rho_{2}$. On the other hand if $\gamma>0, \alpha<0$ and $\beta<0$ we have that energy is transferred from fluids $\rho_{1}$ and $\rho_{2}$ to the cosmic fluid $\rho_{3}$, so the fluids $\rho_{1}$ and $\rho_{2}$ are being diluted due to their interaction with $\rho_{3}$. Let us consider an explicit example of such a scenario. If $n=3, q_{30}<0, C=0, \omega_{3}<\omega_{2}<1$ (note that in this case $\left.\rho_{3}>0\right), \omega_{1}>1$ and $Q<1$ we have that $\gamma<0$, $\alpha>0$. For the other interacting term we have that $\beta>0$ if $\tilde{q}<q_{30}<0$, and $\beta<0$ if $q_{30}<\tilde{q}$, where

$\tilde{q}=-\frac{(1-Q)\left(\omega_{1}-1\right)\left(1-\omega_{3}\right)}{\left(\omega_{1}-\omega_{3}\right)}$.

So we have that energy is transferred from $\rho_{3}$ to $\rho_{1}$ and to $\rho_{2}$ if $\tilde{q}<q_{30}<0$; and for $q_{30}<\tilde{q}$, fluids $\rho_{3}$ and $\rho_{2}$ transfer their energy to the cosmic fluid $\rho_{1}$. It is interesting to note that this kind of triply interacting fluid configurations also has been considered in the framework of the FRW cosmologies (Cruz et al 2008; Jamil et al 2008).

\section{Conclusions}

In this paper we have provided a detailed analysis of Kasner cosmologies dominated by two or three relativistic cosmic fluids. Both cases can be exactly solved in the framework of Einstein field equations. It was shown that the case, where each cosmic fluid evolves separately according to standard conservation laws, leads us to the trivial case of Kasner cosmologies dominated by a single fluid; while if the anisotropic expansion is dominated by cosmic fluids which are not conserved separately (both for two-fluid configurations and at least two for threefluid configurations), then the cosmological scenarios are not at all trivial. For two-fluid cosmological scenarios there exist only cosmological scaling solutions. For three-fluid configurations, among cosmological scaling solutions, there exist also more general ones. It is shown that for two or three-fluid cosmological scenarios, by requiring the positivity of energy densities, there always 
is a matter component which violates DEC in this kind of anisotropic cosmologies.

Finally, let us consider more precisely the general constraints valid for multi-fluid Kasner cosmologies and expressed by Eq.(12) and $S=1$. Constraint (12) implies that for each $p_{i}$ we have that $-1<p_{i}<1$. By using $S=1$, we can replace $p_{3}=1-p_{1}-p_{2}$ into $Q=p_{1}^{2}+p_{2}^{2}+p_{3}^{2}$. Now if we consider $Q$ as a given parameter we find that

$p_{1}=\frac{1}{2}\left(1-p_{2} \pm \sqrt{-3 p_{2}^{2}+2 p_{2}+2 Q-1}\right)$,

and then, in order to have a real $p_{1}$, we must require that $-3 p_{2}^{2}+2 p_{2}+2 Q-1 \geq 0$. Thus the constraint $1 / 3 \leq Q \leq 1$ must be imposed. So the behavior of three scale factors $a_{i}=t^{p_{i}}$ in multi-fluid Kasner cosmologies are restricted to exhibiting decelerated expansions in all three directions, or even contraction in several directions.

\section{Acknowledgements}

The authors thank Paul Minning for carefully reading this manuscript. This work was supported by CONICYT through Grant FONDECYT N ${ }^{0} 1080530$ and by PhD Grants N0 $21070949(\mathrm{FA})$ and N 21070462 (PM). 


\section{References}

Armendariz-Picon, C., Mukhanov, V.F., Steinhardt, P.J., Phys. Rev. Lett. 85, 4438 (2000).

Astier, P. et al., Astron. Astrophys. 447, 31 (2006).

Barrow, J.D., Phys. Rev. D 55, 7451 (1997), Barrow, J.D., Maartens, R., Phys. Rev. D 59, 043502 (1999).

Barrow, J.D., Clifton, T., Phys. Rev. D 73, 103520 (2006).

Bean, R., Magueijo, J., Phys. Lett. B 517, 177 (2001).

Bennett, C. L. et al., Astrophys. J. Suppl 148, 1 (2003), astro-ph/0302225

Bini, D., Cherubini, C., Jantzen, R.T., Class. Quant. Grav. 24, 5627 (2007).

Bini, D., Cherubini, C., Geralico, A.,Jantzen, R.T., Class. Quant. Grav. 26, 025012 (2009).

Bozza, V., Veneziano, G., JCAP 0509, 007 (2005).

Brevik, I.H., Pettersen, S.V., Phys. Rev. D 56, 3322 (1997)

Brevik, I.H., Pettersen, S.V., Phys. Rev. D 61, 127305 (2000).

Cataldo, M., del Campo, S., Phys. Rev. D 61, 128301 (2000).

Cataldo, M., del Campo, S., Salgado, P., Phys. Rev. D 63, 063503 (2001).

Cataldo, M., Cruz, N., del Campo, S., Lepe, S., Phys. Lett. B 509, 138 (2001).

Cataldo, M., Mella, P., Minning, P., Saavedra, J., Phys. Lett. B 662, 314 (2008).

Chimento, L.P., Jakubi, A.S., Pavon, D., Zimdahl, W., Phys. Rev. D 67 083513, (2003).

Copeland, E.J., Niz, G., Turok, N., Phys. Rev. D 81, 126006 (2010).

Cruz, N., Lepe, S., Peña, F., Phys. Lett. B 663, 338 (2008).

del Campo, S., Herrera, R., Olivares, G., Pavon, D., Phys. Rev. D 74, 023501 (2006).

Erickson, J.K., Wesley, D.H., Steinhardt, P.J., Turok, N., Phys. Rev. D 69, 063514 (2004).

Gavrilov, V.R., Melnikov, V.N., Abdyrakhmanov, S.T., Gen. Rel. Grav.36, 1579 (2004).

Goliath, M., Nilsson, U.S., J. Math. Phys. 416906 (2000).

Gunzig, E., Nesteruk, A.V., Stokley, M., Gen. Rel. Grav.32, 329 (2000).

Guo, Z-K., Zhang, Y-Z., Phys. Rev. D 71, 023501 (2005).

Halpern, P., Phys. Rev. D 63, 024009 (2001).

Hinshaw, G. et al. astro-ph/0603451.

Ivashchuk, V.D., Melnikov, V.N., Grav. Cosmol. 14, 154 (2008).

Jamil, M., Rahaman, F., gr-qc/0810.1444.

Kofinas, G., Panotopoulos, G., Tomaras, T.N., JHEP 0601, 107 (2006).

Lee, S., gr-qc/0811.1643.

Mak, M.K., Harko, T., Int. J. Mod. Phys. D 11, 447 (2002).

Nojiri, S., Odintsov, S.D., Phys. Lett. B 639, 144 (2006).

Olivares, G., Atrio-Barandela, F., Pavon, D., Phys. Rev. D 77, 063513 (2008).

Padmanabhan, T., "Understanding our universe: Current status and open issues" in 100 Years of Relativity-Spacetime Structure: Einstein and Beyond, Ashtekar, A., (Editor), World Scientific (Singapore, 2005) pp 175-204; arXiv:gr-qc/0503107

Page, L. et al., astro-ph/0603450.

Pavon, D., Zimdahl, W., Phys.Lett. B 628, 206 (2005). astro-ph/0608636, Percival, W.J. et al., Astrophys. J. 657, 645 (2007).

Pinto-Neto, N., Santini, E.S., Falciano, F.T., Phys. Lett. A 344, 131 (2005).

Ponce de Leon, J., Class. Quant. Grav. 26, 185013 (2009).

Rashid, M.A., Farooq, M.U., Jamil, M., gr-qc/09013724.

Riess, A. G. et al., Astrophys. J. 607, 665 (2004).

Sadjadi, H.M., Alimohammadi, M., Phys. Rev. D 74, 103007 (2006).

Setare, M.R., Sadeghi, J., Amani, A.R., Phys. Lett. B 673, 241 (2009).

Spergel, D. N., et al., Astrophys. J. Suppl 148, 175 (2003), astro-ph/0302209, Spergel, D.N. et al., astro-ph/0603449

Srivastava, S.K., Phys. Lett. B 643, 1(2006).

Svitek, O., Podolsky, J., Czech. J. Phys. 56, 1367 (2006).

Tegmark, M. et al., Phys. Rev. D 69, 103501 (2004).

Tomaras, T.N., hep-ph/0610412.

Zimdahl, W., Pavon, D., Phys. Lett. B 521, 133 (2001). 